\title{
Gravitational Contraction of a Dust Cloud in Space
}

\author{
Alexey. Stanislavovich. Belyaev \\ Department of Physicotechnical, "All-Russia Thermal Engineering Institute" (JSC "VTI") Joint-Stock Company, Russian Federation
}

Copyright $(2016$ by authors, all rights reserved. Authors agree that this article remains permanently open access under the terms of the Creative Commons Attribution License 4.0 International License

\begin{abstract}
Demonstrated here is the previously unaccounted for tendency in the dynamics of the process of gravitational contraction of the dust cloud in space. The article concludes that the mass tends to consolidate on the periphery of the contraction cloud. The reason for an accelerated expansion of the Universe is explained.
\end{abstract}

Keywords Gravitation, Mass Distribution, Large-scale Structure of Universe, Dark Energy

\section{The History}

Despite the colossal pace of development of science, the nature of the relationship of gravitational forces has still not been clearly identified.

Newton identified the force of Earth's attraction with gravitational forces acting in space, and derived the law of universal gravitation (XVII century), in which, instead of using the gravitational masses he uses inertial masses, which appears in the laws of mechanics. However, Newton was fully aware of this. Therefore, he was forced to check his mathematical operations through experiments. With decent precision for his time, he got the result that the amplitude of the swinging pendulum is independent of its mass, which enabled him to conclude that the inertial mass of the pendulum is equal to its gravitational mass (in their physical nature the gravitational force must be proportional to the gravitational masses but the experiment demonstrates their proportionality to the inertial masses of the pendulum). Later experiments conducted by the method of Eotvos showed the independence of the ratio of the inertial mass to gravitational one from the type of substance and its charge, which do not contradict the accepted principle of equivalence of mass. But the reason for the equality of the masses remains unclear. Description of the gravitational field through the law of gravity in combination with the principle of superposition (the exposure of the test mass to the various gravitational objects is added together through a vector summation and do not affect each other) is complete.

In the XVIII century, Coulomb formulated a law of interaction of electric charges, similar to the law of gravity, and in the XIX century, Maxwell used vector analysis to describe the electric and magnetic fields (and then combined these fields). Hamilton laid the basis of vector analysis. He introduced the vector as an alternative to the coordinate notation. Gibbs developed the vector analysis to become an independent part of mathematical physics. To do this, he used the works of Green, Stokes, Gauss and Ostrogradsky. Heaviside gave vector analysis the form it has today. The laws of electrostatics and gravity are identical in structure, therefore, after a successful introduction of vector analysis into electrodynamics, it was inevitably then applied to gravity. The gravitational field and the mass distribution are described by continuous functions of points. The curl of the gravity field intensity vector is equal to zero, therefore, the gravity field is vortex-free. According to the vector analysis, the vector of vortex-free field can be represented as the gradient of a certain auxiliary function called the scalar potential. At the same time the divergence of field vector is a known function of the coordinates. This implies that the Poisson equation can be used to describe the gravitational field (the partial differential equation of the second order). The solution of the equation gives the distribution of gravitational potential. The vector description of gravity provides an alternative to Newtonian description within the framework of classical physics (each approach is independent, but either can be represented as a consequence of the other).

In 1846, Adams and Le Verrier using the law of universal gravitation and taking into account all the required amendments to the disturbances of Uranus' orbit, predicted the theoretical existence of the planet Neptune, which was soon after discovered experimentally. However, the observed shift of the perihelion of Mercury was not described by Newton's law for all the hypotheses of possible disturbances of its trajectory. As a result, the observed shift of the perihelion of Mercury turned out to be the first confirmation of Einstein's general theory of relativity.

Einstein's special theory of relativity, created in 1905, was completely based on the use of the inertial reference systems. This worried Einstein. While reflecting on this subject, he came to the conclusion that a uniformly accelerated reference system with respect to an inertial reference system creates the appearance of the fictitious forces 
characteristically equivalent to the uniform gravitational field. But these fictitious forces are in fact proportional to inertial mass. It becomes possible to naturally explain the principle of equivalence of inertial and gravitational mass (but without explaining the meaning of the term "mass"). To this end, Einstein assumed that the special theory of relativity is valid only where there no gravitational field exists. A generalization was required, in which the special theory of relativity would act as a unique/special case of the new theory. Since the special theory of relativity combined space with time, Einstein had to make a generalization of the geometry of a four-dimensional space-time world within Riemannian geometry, in which the plane Minkowski geometry manifests as a particular case.

A metric tensor of a four-dimensional space-time has ten different independent components. The fourth-order Riemann tensor has twenty independent components for the four dimensions. After coagulating the indices the derived contracted curvature tensor must be equated to zero in order to return to the flat world of Minkowski. During the simplifications one should take into account that significant distortion of flat space are made only by very large masses. Such simplification of the solar system has allowed us to get one independent component for the contracted curvature tensor: gravitational potential. Therefore, in the case of the solar system, Newton's theory can be used with a high level of accuracy. At the same time the planets under their own inertia move around the equipotential surface (gravitational forces do not act on them, they move in straight lines, but in curved space-time). However, strict adherence to Newton's theory is not observed. For the closest planet to the Sun (Mercury) deviations from Newton's theory of gravity become more pronounced. The exact description of the orbits of the planets, including Mercury, in the framework of the general theory of relativity coincides with the orbits observed in practice.

Despite the success of general relativity theory, the interpretation of the essence of gravity began to slowly lean toward the quantum field theory. Today detailed calculations on the description of gravitational fields in the framework of Einstein's geometrical interpretation have almost completely disappeared from literature. A new theory "quantum electrodynamics", based on a constant quantum field theory, was developed in the 1940s. In the 1970s, followed by the development of the theory of electroweak interaction, uniting the weak and electromagnetic interactions into a single quantum field. Such unification of interactions associated with the names of Glashow, Weinberg and Salam. The success of quantum field theory has become so great that almost the entire scientific world has tilted in his favor. Established in the 1980s as part of the Grand Unified Theory, the Standard Model was later verified experimentally by numerous facts (in the Standard Model the strong, weak and electromagnetic interactions are unified). First of all, the discovery of the Higgs boson made using the Large Hadron Collider (LHC) in 2012 should be highlighted. Along the way, it is useful to note that although the Higgs boson seems to be the factor that explains the essence of the concept of "inertial mass" (in reality, many new questions are still arising), it does not exert any power in the question of the equivalence of masses.

The LHC is used to analyze high-energy processes and subtle effects in particle decays of light particles, attempts are being made to find a deviation from the Standard Model for the construction of the New Physics, which includes the gravitational interaction. However, currently there is no direct evidence of physics beyond the Standard Model, except for the discussion of small deviations from the Standard Model. Currently the quantum electrodynamics has won primary scientific position, and quantum field theory is dominant, the gravitational theory, after a preliminary success of the general theory of relativity, is now covered in academic literature without emphasizing the fundamental role of geometry, and paving the path of gravity to the elementary particles. Weinberg and Feynman were the first to choose these policies (see e.g., [1,2]). The main direction of today's quantum gravity is Loop quantum gravity, and the main direction of the New Physics, including a description of its gravitational interactions, is the string theory.

In conclusion of this historic overview one must point out that in order to understand the kinematics of gravitational relations, especially in the case of medium gravitational fields and velocities, it is not necessary to use current physics modes of gravitation. It is enough to follow the schematic solutions within the framework of classical gravitational, which is what this paper does. Moreover, high speeds and gravitational potentials are not enough to skew the conclusions based on the schematic solutions, unless we are talking about concrete calculations.

\section{The Problem}

Since the gravitational field is vortex-free, it can not contain closed lines of force (masses are the sources of field lines in the gravitational field, which go to infinity). This fact clearly indicates the validity of the Gauss theorem, according to which the flow of the vector of tension of the gravitational field through any closed surface is proportional to the mass contained within the volume defined by the surface.

The Gauss theorem is consistent with the law of universal gravitation if the test gravitational mass is positioned outside the selected closed surface which highlights the volume and the considered mass. For example, it becomes very clear why the forces of gravity in the vicinity of the Earth's surface act as if this surface is not there, and the whole mass of the Earth is located in its center of mass.

However, Gauss's theorem shows that for a spherically symmetric mass with a concentric spherical cavity in the middle, the strength of the gravitational field in the cavity is equal to zero. In other words, the gravitational field inside a sphere with its mass uniformly distributed over the surface is non-existent. If the mass is not distributed evenly around the 
circumference of the sphere, but only in the areas around the opposing poles, then the polar zone segments will be attracted to each other as two point masses located at the mass-centers of their segments and equal to their masses. As the area of the segments grows so will their masses and their centers of mass will converge. Therefore, when there is an infinitesimal gap between the two hemispheres it is logical to expect the maximum force of gravitational interaction. However, according to the Gauss's theorem the power/force lines will already be unable to penetrate into the interior of an almost complete sphere, i.e., the hemispheres attraction to each other at an infinitely small gap must become zero. After a the two hemispheres join into sphere in its internal volume the two test masses will be unable to gravitationally interact not only with the internal surface of the sphere, bearing the distributed mass, but also between themselves. In this case, it seems to be an obvious violation of logic and common sense. For example, a similar effect is not possible within the framework of the quantum theory of the gravitational field. Furthermore, the general theory of relativity, as opposed to Gauss theory indicates a presence of a gravitational field inside a spherical cavity. It is obvious that the resulting output of vector analysis theory for these boundary conditions is incorrect and requires revision.

One would argue that there is no point in dwelling on such a nuance, after all, no one is trying to shield the gravitational field by placing a research laboratory inside the material shell. However, the inaccuracy that is now entrenched in science has started to cause confusion in the understanding of the processes around us and made us perceive them as a doctrine. For example, it is assumed that the gravitational field strength increases linearly from the center of the Earth to its surface. Moreover, the field strength at any selected point on an arbitrary inner surface of the sphere does not depend on whether or not the outer mass (crust) that surrounds the chosen field is mentally disregarded. At the same time no attempts are made to explain whether any gravitational potential can exist in the inner sections of the Earth using the chosen approach to the explanation of the gravitational field. Another good example is the complete disregard for the influence of the invisible galactic halo on the movement of the stars, the scientists seem to not even suspect that the aphelion elliptical orbit of the stars may be biased due to their approach to a large foreign mass.

This paper discusses the application of the theory of gravitation associated with spontaneous gravitational contraction of dust clouds in space. Currently, it is believed that in the process of contraction of a dust cloud its outer layers cannot influence the dynamics of the inner layers. Below it is shown that, in contrast to vector analysis, the law of gravity in combination with the principle of superposition clearly indicates the presence of a gravitational field inside a closed sphere while its mass is distributed over its surface. However, this conclusion is symbiotically related to the fact that the presence of a gravitational field in the internal spherical cavity with central symmetry cannot not influence the mass located within the cavity.
Gravitational forces clearly manifest themselves in space where they prevail. Therefore, consideration of the gravitational interaction automatically applies to cosmology. At the same time all the gravitational effects are studied for systems placed in outer space (no friction forces). The adopted model in cosmology and the resultant physical processes are well described in $[3,4]$.

This paper explores the mechanical movement with the gravitational interactions, i.e., the discussed material does not only apply to cosmology, but also to classical mechanics. The resulting cloud compression scheme that has been refined based on the resultant dynamics has many important consequences, but the main conclusion indicates the absence in the universe of dark energy of unknown nature.

\section{Materials and Methods}

If you place two objects into a vacuum, fixed relative to each other in an inertial frame of reference, then under the influence of gravitational forces of attraction, they will begin to move towards each other along a straight line connecting their centers of mass, until they collide at their common center of mass. The presence of a velocity, directed along the line joining the centers of mass, does not change the principal idea of the interaction, and the interaction remains the same: the objects are moving along the same straight line due to the attraction to the center of mass of the system. In this case, there is a possibility of a change in the direction of movement of one or both of the primary objects to the opposite, whereupon objects will eventually inevitably collide. It is also possible that one or both objects, if the distance between them is growing, will be unable to change the direction of their movements, and the distance between them will grow indefinitely. But this divergence will be happening with a definite reduction in the rate of divergence.

If the objects at the initial time have randomly directed velocities, the resulting interactions will be drastically different from those described above. In order to be able to describe the motion of such a system, we would need to use the angular momentum conservation law. The trajectory of interacting objects, in this case, would be curved. A distinctive feature of these initial conditions is the possibility of capturing the effect the gravitational fall of one object onto another in stationary trajectories of their motions. For example, the elliptical orbit of the planet around a star which is at the center of the ellipse, in the absence of friction is able to persist indefinitely (the kinetic energy of the planet will transform into its potential energy and vice versa, and the total energy of the planet, in the absence of any losses, will remain constant). In this case, the planet's velocity component, which is perpendicular to the major axis, is at its maximum and equal in magnitude at the apexes of the major axis, it then decreases in magnitude for all other points of the elliptical trajectory and reaches zero at the apexes of the minor axis. In contrast, the planet's velocity component, which is perpendicular to the minor axis, is equal to zero in 
the apexes of the major axis, and it has the maximum and equal in magnitude values at the apexes of the minor axis. The simplest observed case of gravitational convergence is the motion of a satellite in a circular orbit where the object that attracts the satellite does not perform any mechanical work on it (the satellite's orbital velocity does not change in magnitude, but its direction is perpendicular to the gravitational forces at all points of the trajectory). According to the general theory of relativity, it is in such cases that the satellite's motion takes place on an equipotential surface (for the source of the gravitational field with central symmetry in the mass distribution).

If we generalize the enlarged scheme of evolution, such as that of galaxies, in which the motion of the components of space objects that make them up is happening with energy loss, then, at the first glace, one might suggest the following: in the case of chaotic directions of velocity the component of the galaxy, at the initial point in time, has a disordered structure (irregular galaxies), which gradually transform, first, into a helical (spiral galaxies), and then into elliptical structures (elliptical galaxies; the closer the orbits of the objects come to the center of the galaxy, the closer their shape becomes to a circle). However, observations show that these assumptions are incorrect: all galaxies, irrespective of their structure, are similar in age. This is due to the fact that the processes of the internal evolution of galaxies are very complex and are continuously accompanied by the birth of new generations of stars. But the structure of galaxies, star systems and galactic clusters do have one thing in common: the closer you get to the center, the greater the accumulation of mass. This happens because when objects rotating around the common center of mass experience energy loss they tend to reduce their orbital radii (in spite of the energy losses, the average speed of objects with decreasing radii of rotational orbits increases).

Consider the process of gravitational contraction of a dust cloud in space. From now on, the term dust cloud shall refer to a cloud the constituent elements of which are able, while colliding with each other, to unite into composite structures or generate a new type of element. The main feature of the considered cloud is the consolidation of masses of its colliding parts. Moreover, since we are talking about clusters of electrically neutral space objects, this study only focuses on the gravitational interactions in the absence of other long-range forces. In particular, a cluster of galaxies, and even the Universe as a whole, can be roughly considered a dust cloud, in which galaxies are dust particles. In the process of collisions the galaxies, under certain conditions, might be combined into a new element of a specific physical system. Of course, it is unacceptable to take galaxies as elementary points when studying the acts of their collisions, when the colliding galaxies can not only form a coherent system, but also scatter, with possible changes in their masses.

The current study of the contraction dynamics of elements of dust clouds leads to quite unexpected results. It shows that in the process of contraction the density of matter inside the cloud has a different special distribution compared to the final results. The examined mechanisms of contraction allow us to understand the reasons for the accelerated divergence of galaxies. Furthermore, the transference of the obtained results into an analysis of gas-dust clouds contraction will become useful for describing the mechanism of hard element separation, in the formation of planetary systems.

In order to understand the essence of the gravitational interaction and simplify the argument, it is first convenient to consider the system with its elements being at rest relative to each other in the initial moment in time. Let us imagine a chain of identical balls, situated on one straight line and equidistant from each other at the distance of R. Around the chain of the interacting balls the resultant gravitation field will be formed by vector summation of gravitational fields of each ball. Each ball in the chain will be influenced by resultant forces of mutual gravitational interaction of the balls, and all these forces will appear to be directed towards the central ball. The forces acting upon the outermost balls will be equal to each other in magnitude, but opposite in direction. For the central ball the resultant force of gravitational interaction will be equal to zero. For all intermediate balls the magnitude of the forces will decrease towards the center. The proposed illustration of the distribution of gravitational forces along the chain of balls is simple and does not require additional comments or calculations. The obtained result of gravitational contraction is reflected in Fig. 1A.

At the initial point of time, i.e. at the quiescent state of the balls relatively to each other, the force applied to the $\mathrm{j}$-numbered ball will be defined by expression:

$$
F_{j}=\frac{G m^{2}}{R^{2}}\left\{\sum_{i=1}^{n-j} \frac{1}{i^{2}}-\sum_{i=1}^{j-1} \frac{1}{i^{2}}\right\}, \quad 2 \leq j \leq(n-1),
$$

where $n$ is the amount of balls, $G$ - gravitational constant, $m$ - the masses of the balls, $R$ - the initial distances between the balls.

For a better demonstration of the result, which will be achieved by considering the contraction of the chain of identical balls, let us assume that the balls, as the result of colliding with each other, interflow, "sewing" each other throughout, without extension of the size of a newly formed ball (they join into a ball with a double mass, density and previous center of gravity). Galaxies behave in a similar fashion during head-on collisions while permeating each other and combining their stars, gases, planets and asteroids.

As the countdown begins, the chain will tighten, and will do so unequally: the balls will no longer be equidistant from each other, they will move with a large acceleration the closer to the periphery they get. As a result of the balls movement, the proportions of forces will be redistributed. Ball No. 1, will approach ball No. 2 faster compared to all the other balls and will begin to interact intensively with ball No. 2 and with the rest of the balls, which, along with the increasing of force $F_{1}$, will cause a decrease of the rate of 
growth of force $F_{2}$, and all the rest of the forces $F_{j}$ in the direction of the central ball (to simplify, we can note that none of the other balls, except for ball No. 1, have had time to move: this simplified version will help towards easier interpretation of the situation). The described trend will not only remain but also increase: by the time the balls No. 1 and 2 unite, the distances between the rest of the balls will continue reducing, and the closer the balls are to the edge of the chain, the more intensive will the process be, i.e. the distances between the periphery balls will consistently reduce at a faster pace than the distances between the balls in the chain towards the central zone, and the mass of the outermost ball will grow constantly.

In some period of time a certain quantity of periphery balls will unite, and in periphery zones the forces influencing the balls, will change their direction (Fig. 1B). In another period of time all the forces will change their direction and will be directed not towards the central ball, but towards the edges of the chain (Fig. 1C). Meanwhile, the resultant force of gravitation will remain zero during the whole process of the uniting of balls, for the central ball, and at the periphery the zone of increased density of the balls will be formed.

\section{Preliminary Results}

In the upshot, we have a very interesting result. It is obvious that all the balls will gather into one united ball in the center. But the fact that the balls, aspiring to unite, will move most of the time not towards the center of the masses of the balls system, but, on the contrary, to the periphery of the chain, is an unexpected result, conflicting with the formed traditional views. But with a more careful studying of the dynamics of changing the forces of interaction between the balls, the conclusion obtained becomes obvious and irrefutable. In general, the whole chain continuously contracts, but internal balls of the chain begin to be decelerated, and finally, in a certain period of time, change the direction of their movement, creating an increased concentration at the periphery. The movement of internal balls from the center happens against the background of simultaneous growing of masses of boundary balls, moving towards them. While this, the mass centers of two groups of balls (on the left and on the right from the central ball) move towards each other symmetrically relatively to the center of mass of the whole system of balls.

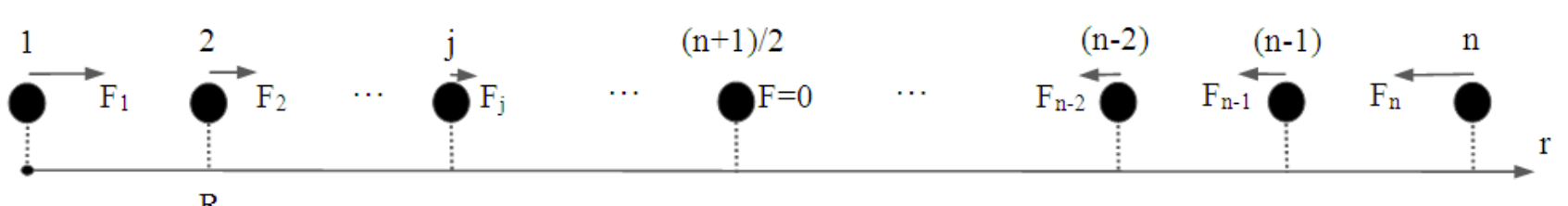

A

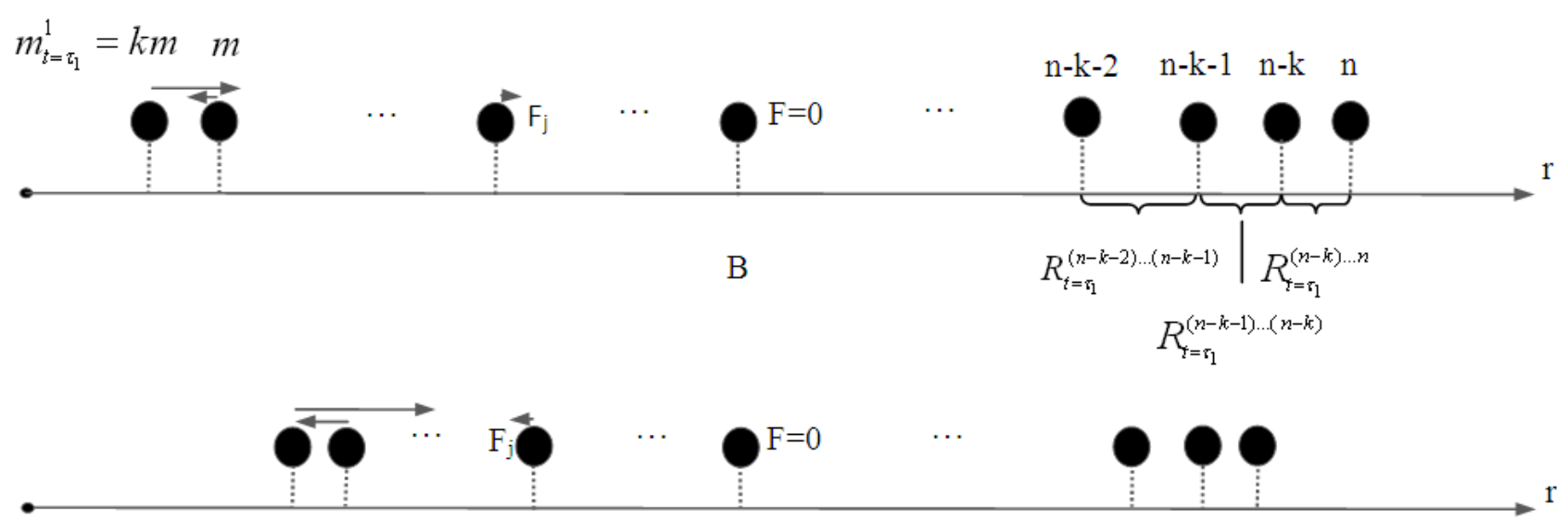

$\mathrm{C}$

Figure 1. The scheme of contraction under the influence of gravitation forces of a chain of a finite number of balls:

Part A. The initial point of time $t=\tau 0$ : to show the correlation of magnitudes of forces, their vectors are conventionally depicted above the balls but don't come from the centers of the balls.

Part B. The point of time $\mathrm{t}=\tau 1$ : a part of balls acquires acceleration of the opposite direction, the equality of distances between all the balls is damaged $\left(R_{t=\tau_{1}}^{(j-2) \ldots(j-1)}{ }_{\neq} R_{t=\tau_{1}}^{(j-1) \ldots j}\right.$ ), the outermost balls from each side of the central ball have had time by the moment $\tau 1$ to absorb (k-1) balls each, and their masses have been increased by $\mathrm{k}$, the masses of all the rest balls remained unchanged, equal to $\mathrm{m}$.

Part $\mathrm{C}$. The moment of time $\mathrm{t}=\tau \mathrm{i}$ : all the balls, except for the boundary ones, have changed the sign of their acceleration (the acceleration of the central ball remained zero). 


\section{Generalization of the Results}

The preliminary result is obvious, but it was obtained for a one-dimensional model. If we take a simplified three-dimensional diagram - if we aggregate the chains of balls, the central balls which are the same, and the rest of the balls lie on a sphere whose centers coincide with the center of mass of the central sphere, the situation will not change: the higher density of the balls during the compression process will be observed on the periphery, the lower density - in the central zone.

This is as obvious as it is for a one-dimensional chain. New gravitational forces of the new balls that did not appear in the one-dimensional model will begin to exert further influence on each ball considered in the one-dimensional chain due to superposition. Due to the central symmetry of the spatial distribution of mass, the projection of new gravitational forces directed perpendicular to the axis on which the balls are all located in a chain, will have a mirror symmetry about the axis and will mutually compensate each other. No special calculations for an explanation of this effect are required. The projections of the additional forces directed along the axis of the chain, after a vector summation will be directed to the central ball and their quantities will be distributed in the same proportion as the gravitational forces are distributed in a one-dimensional model. Thus, the resulting picture remains exactly the same, except for the appearance of extra mass, forcing all the separate chains, passing through the central globe, to gather at a higher rate. Thus, the three-dimensional dust cloud, with zero relative velocity of its constituent elements in the initial moment of time, in the process of its compression must thicken its peripheral layers. It is obvious, that it is not necessary to demand central symmetry for the initial distribution of a fixed mass in this scheme of compression. The result of the dynamic distribution of mass in the process of spontaneous contraction would unquestioningly remain the same.
But everybody has been used for a long time to the fact that in the center of a collapsing object the temperature and the density of matter is always the biggest. This is a good reason to analyze this question in a little more detail.

Let us consider the aggregate of not big, equal in mass balls with masses $M$, the mass centers of which are on one sphere with an equal pitch of location. The sum of the masses of the balls is $M_{\Sigma}$ It is obvious that each ball is influenced by gravitational force of the resultant interaction with the rest of the balls, directed towards the center of the sphere: the forces of in-pair gravitational interaction of a ball with the mass $M$ with each of the rest of the balls of the aggregate individually give projections on the axis, led through the center of mass of the ball, and the center of mass of all the balls with mass $M_{\Sigma}$ (the center of the sphere) with one direction towards the center of the sphere, and on the axis, perpendicular to the first axis - of the different sign, besides, owing to the symmetry of the balls location and the equality of their masses, compensating each other in total. If, apart from the gravitational forces, there are also short-range repulsive forces between the balls, for example, the balls are solid and do not deform, then the aggregate of the considered balls at a particular point in time will lock into a spherical shell (Fig. 2).

If we place a trial body with mass $m$ near the considered cover, it will be attracted to the cover, both in the case of placing the trial body out of the cover, and placing it inside the cover. For the trial body, placed outside, the resultant gravitational force of interaction with all individual balls will be, similar to the described process of evaluation of resultant gravitation of the balls of the aggregate, also directed to the center of the sphere (to the center of masses of the aggregate of all the balls), and the result of the interaction will be as if the trial body with mass $m$ interacted with a dotted body with mass $M_{\Sigma}$ situated in the center of masses of the balls group.

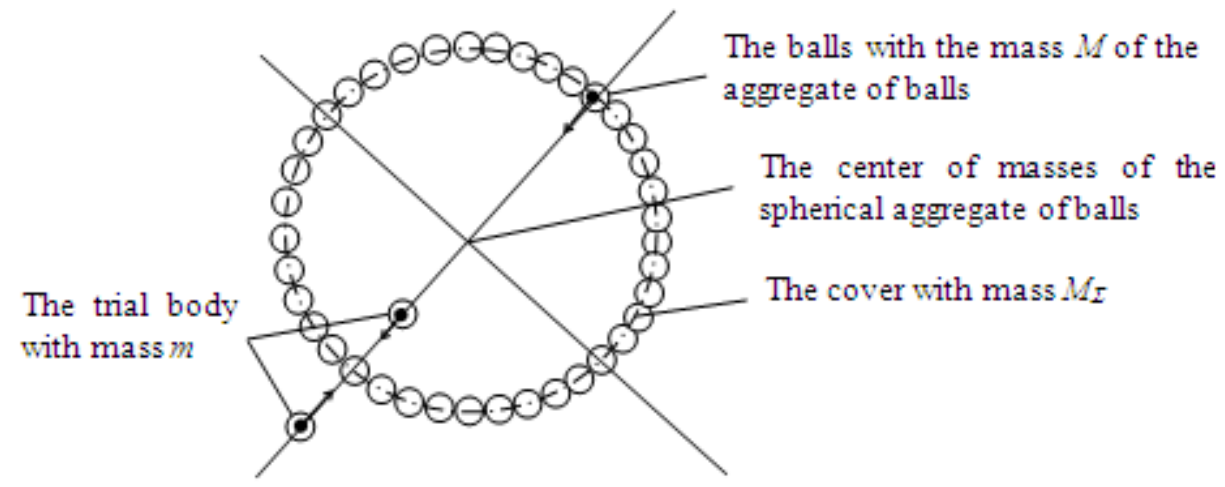

Figure 2. The scheme of directions of resultant gravitation forces of the aggregate of balls, situated on the sphere of balls 
For the trial body, placed inside the cover, the projections of forces of gravitational interaction on the axis, going through the center of masses of the trial body $m$ and through the center of masses of the group of balls of the cover (the center of the sphere), will be different in sign, but the contribution of vectors, directed from the center of the sphere, will be more essential. And the projections of forces on the perpendicular axis will compensate each other. Correspondingly, the resultant force of gravitational interaction will be directed along the diameter to the surface of the sphere (perpendicular to the tangent of the sphere, i.e. as if perpendicular to the internal surface of the sphere). The closer the trial body is to the center of the sphere of the cover, the less force of gravitation influences it, and in the center of the sphere the trial body will be in imponderability. It is obvious that the force of gravitation of the trial body to the surface of the cover from inside will be less than the force of gravitation of the same trial body to the surface of the cover, but located outside the cover. Deriving the formulas of the analytical calculation of values of gravitational forces inside the cover according to the distance from the trial body to the surface of the cover doesn't meet any principal difficulties, but is beyond the present article. It should be highlighted that the law of gravity in combination with the principle of superposition in this specific case does not correspond to the Gauss theorem of vector analysis. Inside the cavities of massive objects the gravitational field is inevitably present.

If we fill the sphere, from the above example, with the same balls with masses of $M$, the resultant forces of gravitation, influencing these balls, will appear to be directed to the center of the sphere, but their magnitude will decrease as they approach the center. This reasoning clearly testifies that, in the presence of only forces of gravitation, the contracting accumulations of matter have to have the least density in the center of the accumulation and the biggest at the periphery. In fact, the periphery elements of the aggregate are influenced by all the rest of the elements towards the center of mass of the aggregate, i.e. all the mass of the aggregate works in one direction, that's why the resultant gravitational force, directed to the center, is maximal. However, the closer the element of the aggregate is to the center, the more it is influenced in the opposite direction from the center by the periphery elements of the aggregate. If in the aggregate of matter we place a multitude of absolutely rigid imponderable spherical skeletons, on which spherical covers from the matter with thickness of layer $d r$ lean (situated on the outer side of load-bearing skeletons), the maximal density of the matter of covers will be at the periphery, and the minimal - in the center. But the skeletons, preventing the matter from moving towards the center, as a matter of fact, mean the introduction of additional forces of repulsion into the system. And the notion of density itself is directly connected with the existence of forces of repulsion. The forces of repulsion cause the appearance of other characteristics of the aggregate, such as pressure and temperature. In reality, absolutely rigid skeletons don't exist, and usually particles of the matter of the upper layers of the aggregate transfer their pressure to the lower layers (including the case of gas clouds with Brownian movement). But the mechanism of transferring of the pressure to the deep layers is connected exactly with the appearance of forces of repulsion. That's why the density of the matter and its pressure in the center in practice is always bigger than at the periphery. The pressure and density in the center will grow both in case of a crystal, huge in size, and of a huge drop of liquid, and gas.

Thus, the main reason of inversion of the density distribution is arising of the forces of repulsion. To balance the force of gravitation $F_{j}$ (see Fig. 1A), it is necessary to apply the force of repulsion $-F_{j}$ from ball $(j+1)$ to the ball $j$. But, according to the third Newton's law, the ball $(j+1)$ will be influenced by the corresponding force of counteraction $+F_{j}$, i.e., the ball $(j+1)$ in the direction of the center will be influenced by the force $\left(F_{j+1}+F_{j}\right)$, which, in its turn, needs to be balanced by the ball $(j+2)$. Such balance is established on the two sides along the whole chain and finally determines increased densities in the central zone.

In the scales of the Universe while considering interaction of galaxies between each other and different objects inside the galaxies the force of gravitation prevails, that is why the obtained result is relevant for understanding the evolutionary processes of the Universe. If we consider the Universe as a whole, the gravitational interaction of galaxies will differ from the considered model, firstly, because of the availability of galaxies' speeds at the initial point of contraction, directed from the center of mass of the Universe. It should be emphasized that velocities are not randomly directed, but along the radius of the expanding universe. Thus, the change of the initial conditions will not lead to any principal changes of the results of the contraction forces (gravitation) on the balls-galaxies, and will only influence the dynamics of the process. If the speeds of the periphery galaxies at the initial point of time exceeded the second space speed, they will not change the direction of their movement. The fact that the galaxies are not formed at once, does not change the obtained results, the basic idea is still the same. The conclusion about the accumulation of matter at the periphery cannot and will not change due to the existence of the initial radial velocities of galaxies and the evolutionary development.

If we don't arm ourselves with the new abstract notion of "dark energy", it is now impossible to say whether our Universe expands (whether the diametrically opposite periphery matter moves away from each other) or not, and if it does, whether it will continue to expand in the future, or the expansion will be replaced by contraction. For this, additional initial information is required. But in any case, internal galaxies of the Universe will accelerate towards the periphery due to joint gravitational interaction of all the matter of the Universe. We need to understand that red shifts of galaxies are habituated not by the expanding of the Universe, but by the work of gravitational forces. The Universe itself could have contracted a long time ago. And the accelerated divergence of galaxies is habituated not only 
by dark energy, which simply does not exist, but also by the work of gravitational forces.

Thus, the analysis made above for our Universe enables the following conclusions:

1. The main density of matter is concentrated at the periphery of the Universe.

2. The movement of most of the galaxies has to happen with acceleration in the direction from the place of birth of the Universe (thereafter, an observer from the Earth, not depending on the Earth's location relative to the place of birth of the Universe), besides, the further is the location of the galaxy from us, the more is its acceleration while moving away.

\section{Discussion}

The impetuous processes at the periphery of the Universe testify to the ideas represented above. These processes have been found out with the discovering of quasars. Quasars are characterized by: huge red shifts; luminosities, exceeding the luminosities of galaxies thousands of times; sizes, millions of times smaller in size than galaxies (sizes were estimated by astronomers by variability of radiation of quasars); quick and slow fluctuations of brightness. Besides, quasars are not explosive, in general stable and long-existing objects. The mass loss of a quasar with radiation can be estimated by their luminosity, but estimation of mass of the quasar itself will depend on the mechanisms of radiation. In any case, the mass of a quasar will by many orders of magnitude exceed the Sun's mass, will be comparable or some orders of magnitude less than the mass of the galaxy, and in size the quasar will be closer to a stellar system, than to the galaxy. The spectrum of radiance of quasars is very wide and monotonous in the power of radiance, it is much different from Planck's spectrum, the largest powers correspond to ultraviolet, roentgen and even gamma radiations. There are wide and narrow lines of linear emissive spectrum. The types of chemical elements identified by emissive lines indicate the impossibility of joint co-existence of identified ions in the same conditions. The lines of absorption are few. Thus, all observations indicate the complex structure of quasars (the observations also testify to the complex structure of other radio galaxies). Almost all modern models of quasars require the presence of either high star density in the center of the galaxy, or a huge amount of gas, or, more preferably, both of these together. The ideal conditions for the accumulation and warming up of galactic gas and increasing of star density are created by the collisions of the galaxies, particularly, if these collisions are repeated. The observed quantity of quasars gradually grows with increasing $z$, where $z$ is Doppler displacement (although, it drops dramatically almost down to zero while $z \approx 3.5$ ), which confirms the described model of contraction of a chain of united balls. Quasars could be driven to such high speeds (red shifts) only by gravitational forces of all the matter of the Universe. Quasars are at the periphery zone of the Universe. But it doesn't mean, that beyond the limits of quasars, which still have peak values of red shift, there is no matter. As spectral red shift of the "outermost" matter can be either insignificant or even violet, and the distance to it is maximal. Furthermore, beyond any doubt, the "outermost" matter doesn't generate any electromagnetic radiation at all, just like an atomic nucleus, or an isolated or separated from matter neutron star.

The distance to faraway objects, to which the quasars are also attributed, are nowadays measured by red shifts of their radiation and are calculated with the Hubble law. That's why the result was that objects with red shift of more than about 0.5 radiated their stream of light, by which we define the red shift, before the formation of our solar system. But the closer to the periphery of the Universe is the galaxy, the more intensively the Hubble law is broken for it. So it is incorrect to think that spectrums of quasars are characteristic for the evolutionary past of the Universe. Quasars don't show us the period of birth of the first stars and galaxies.

The existing theories of formation and developing of galaxies are not able to explain the phenomenon of quasars as the ancestor of a galaxy. Besides, the emissive lines of the linear spectrum of a quasar indicate that it doesn't consist of stars of the first generation and includes heavy chemical elements. Taking quasars as the emissions of black holes, as it is recommended by contemporary interpretations of the phenomenon "quasar", is also quite difficult, because the number of issues, requiring to be solved, is not coming down, but, on the contrary, is growing.

Moreover, to emit radiance from such faraway past to be noticed from Earth, a space object has to be in time to such an area of space, from which light will further go to an observer on Earth with velocity of light at the required moment of time, i.e. in the modern epoch. But it has been a long time since electromagnetic radiation of the epoch of birth of the first stars and the first galaxies left irrevocably the outskirts of the solar system. As the sizes of the cloud of matter, from which the galaxies began to form, in any case were not more than the sizes of the cloud if it expanded with the velocity of light. And as the period from the moment of the birth of the Universe to the moment of the birth of galaxies (the velocity of which is less than the velocity of light) is less in magnitude than the period from the moment of the galaxies' birth to nowadays, all the electromagnetic radiation from the galaxies of the epoch of their birth has left the boundaries of the zone, where today the matter of the Universe is concentrated.

Thus, quasar does not prove that evolutionally quasars appeared first in the Universe and only then did Seyfert galaxies, BL Lac objects, other radiogalaxies and galaxies with weak activity of their nuclei appear. In spite of the fact, that, the light from more remote objects takes more time to overcome the distances to the earth observer, quasars with large Doppler shifts are not an earlier stage of the state of the Universe, and do not come into the state, typical for quasars with less shift. We cannot conclude that initially a lot of quasars with bigger brightness were born, and then their amount decreased with a simultaneous decrease in brightness. 
The real situation is diametrically opposite. Quasars with moderate red shifts would eventually lose their activity, and while remoting from us they would become identified with more difficulty, their amount, fixated in observations, with the increase of magnitude of red shift would decrease. But high density of the matter at the periphery of the Universe and the accelerated movement of matter towards the periphery lead to "warming up" of quasars and an increase in their amount.

\section{Conclusions}

Thus, the accelerated expansion of the universe finds an undeniable and unquestionable explanation in classical gravitation. Therefore, if we consider the principle of Occam's razor, it is unacceptable to introduce "Dark Energy" as a new category of science. This action greatly hinders the development of science.

Furthermore, it should be noted that the above principle of the process of contraction of a dust cloud in space is actively involved in the processes of formation of planetary systems in the gas and dust clouds, but is complicated by accretion accompanying the contraction process, and stellar winds that form stars. However, this topic is beyond the scope of this article and will be discussed in a separate article.

\section{REFERENCES}

[1] S. Weinberg. Gravitation and Cosmology: Principles and Applications of the General Theory of Relativity, Mir, Moscow, 1975.

[2] R. P. Feynman, F. B. Morinigo, W. G. Wagner. Feynman Lectures on Gravitation, Yanis-K, Moscow, 2000.

[3] D. S. Gorbunov, V. A. Rubakov. Introduction to the Theory of the Early Universe: Hot Big Bang Theory, World Scientific Publishing Company, 2011.

[4] D. S. Gorbunov, V. A. Rubakov. Introduction to the Theory of the Early Universe: Cosmological Perturbations and Inflationary Theory, World Scientific, Singapore, 2011. 